\title{
A shell like kilometer spaced array or a honey comb one around Icecube
}

D. Fargion, ${ }^{a, b, *}$ P.G. De Sanctis Lucentini ${ }^{c}$ and Maxim Khlopov ${ }^{d, e, f}$

${ }^{a}$ Physics Department Rome University 1, P.le A. Moro 5, 00185, Rome, Italy

${ }^{b}$ INFN Rome1, P.le A. Moro 2, 00185, Rome, Italy

${ }^{c}$ Physics Department, National University of Oil and Gas Gubkin University 65 Leninsky Prospekt, Moscow, 119991, Russia

'Centre for Cosmoparticle Physics "Cosmion"

National Research Nuclear University ”MEPHI”, Kashirskoe Sh. 31, Moscow 115409, Russia

${ }^{e}$ Université de Paris, CNRS, Astroparticule et Cosmologie, F-75013 Paris, France

${ }^{f}$ Institute of Physics, Southern Federal University, 344090, Rostov on Don, Russia E-mail: daniele.fargion@fondazione.uniroma1.it, desanctislucentini.pg@gubkin.ru

\footnotetext{
${ }^{*}$ Presenter
} 
Icecube neutrino detector a cubic kilometer in South Pole, traces energetic TeVs-PeVs neutrino signals by their cascades or better by their tracks inside its icy volume. Cascades, explosive spherical showers in icy, are mostly for electron or tau or neutral current, tracks are made by muons. High energy tau tracks might show a birth place cascade and a second decay tau shower, allowing a peculiar directional track. In general hundreds TeVs-PeVs Cascades show very poor directionality. Therefore they are nearly blind to disentangle the source event. Therefore we suggest to neglect such cascades in future Gen-2 Icecube. Indeed the most interesting signals are the tens TeVs up PeVs muon upgoing tracks, either born inside, HESE, or outside Icecube: the trought going muon tracks. These ones are more abundant at ultra high energy (UHE) regimes because their interactions is outside the Icecube, mainly in the wider volume of dense rock around and below the Icecube volume. Such several kilometers tracks offer the most energetic and precise neutrino astronomy. Moreover highest tens $\mathrm{TeV}$ or PeVs neutrinos become opaque to the Earth radius. Therefore they are arriving mainly as up-going nearly horizontal tracks, because they are crossing only short Earth cords. In consequence we suggest an upgrade Muon Icecube detector by a widest kilometers spaced concentric spiral array rings (around old Icecube), each arm nearly a kilometer far from the other, leading to the largest and widest array net (for any given string number). Such wider (km) empty array volumes, like a sponge, may better amplify, almost quadratically, the observed mass volume, in comparision to a more dense but homogeneous volumetric cubic one (at 240 or 330 meters distance among strings) as the Icecube Gen-2 project. In our first approximation one may obtain an increased cubic volume reaching about 50 times the kilometer Icecube array, by a shell like spiral volume or a honeycomb cell net, leading not to a few rare (hundred $\mathrm{TeV}$ energy) event a year but to many tens or even a hundred of such neutrino tracks each year, running toward a more statistical rich Astronomy

$37^{\text {th }}$ International Cosmic Ray Conference (ICRC 2021)

July 12th - 23rd, 2021

Online - Berlin, Germany 


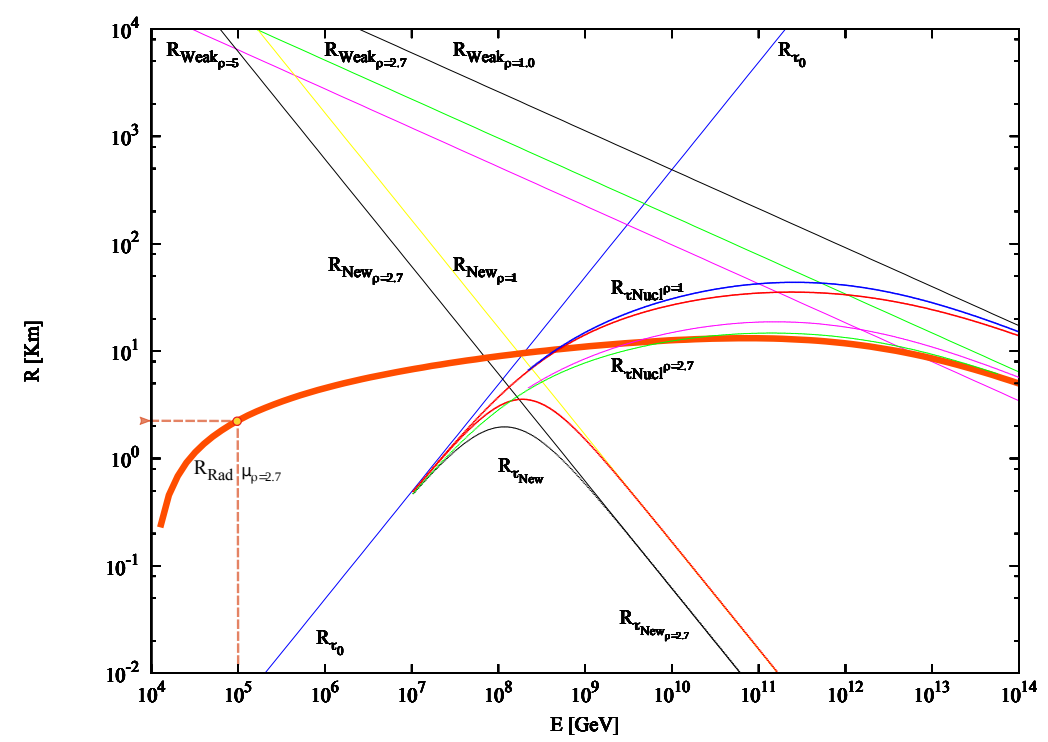

Figure 1: The energetic muon track growth in ice or water is nearly three times than the rock one. In the figure the muon track ( as well as the corresponding tau lepton track) is shown. Above hundred TeV energy the muon track in ice overcome ten kilometer range. Therefore, the associated ideal detector array may be spread its array in large extreme km empty sizes with wide surrounding string frames.

\section{Introduction}

Since nearly a decade (2013) Icecube discovered a peculiar flavor change in tens TeV energy, suggesting a novel, expected, Neutrino Astronomy: [1]. Indeed, while in Icecube at TeVs energies neutrino muons overcome (by a factor twenty) the electron ones, above $30 \mathrm{TeV}$ s the cascade events , (made by electron, neutral current, tau interactions), seemed to suddenly exceed by a factor 2-3 the muon ones. This signature could be a new neutrino Astronomy,(or an additional atmospheric charmed noise [2]). Anyway a different and more clean neutrino astronomy may rise soon by tau air-showers, considered since more than two decades [3]., from mountains [4] or valleys, from ground or space [5, 6] , and searched in more details [7] in different ways in space [8] and on Earth [4]. The airshower by upward tau neutrino may shine at such altitudes where the geomagnetic bending play relevant role, [9]. Therefore future setection need peculiar vertical or crown arrays. Also a new and an exciting neutrino astronomy may rise within the gamma ten-tens $\mathrm{TeV}$ Moon shadow in square kilometer LHAASO or in present or future AUGER and widest areas GRAND arrays.

\section{The TeV-PeV muon track ranges}

The muon tracks induced by upgoing or horizontal UHE neutrinos, with future upgoing tau airshower, are a main key to a sharp neutrino astronomy. Indeed:

1. Cosmic rays, $\mathrm{CR}$, from GeVs up to PeVs energies, are smeared and diffused on our sky leading to the huge downward muon rains and a tiny upgoing neutrino signals, mainly up to 
$10^{13} \mathrm{eV}$, just secondaries of the same muon rains in the opposite side of Earth: the upgoing muon neutrino atmospheric noise. At such highest energies, pions may still decay in flight in atmosphere, but muon are not able to decay. Therefore an atmospheric high energy muon neutrino (made by pions decay) became overabundant over the electron ones (not longer made by muon decay) above hundreds $\mathrm{GeV}$ energies. TeV muons in high and higher energy ranges leave in Icecube long tracks, kilometer long, while electrons, ( suppressed by a factor 20 respect muons), tau and neutral current ones, arise mainly as cascades: spherical showers of lights. Above several tens TeVs energy Icecube discovered since 2013 a remarkable increase in cascade rate comparable or even more abundant than the corresponding muon tracks. Such a revolutionary [1] behavior in flavor change favored the interpretation of a new astrophysical origin. Let us mention that also a charmed atmospheric pollution might also (in principle) solve the flavor change or to be an additional component [2]. The present popular understanding, on the contrary, consider the meaning of Icecube signals as astrophysical in nature.

2. Incidentally the presence of UHECR anisotropy and the few hot spot was also suggesting an eventual connection between the UHE neutrino clustering and the new born correlation with eventual UHECR spot. The earliest far clustering of far redshift UHECR were also suggestive to be relic trace of cosmic, dark neutrino mass halo hit by $\mathrm{ZeV}$ neutrinos [10], [9]. This search of $v$ and UHECR connection at the moment it is not yet conclusive [11],[12]. However we already noted and underlined the presence of a very relevant galactic component in UHECR EeV anisotropy map energy from Crab, Vela and LMC and SMC. [13]. In this search for correlations we concluded from the UHECR clustering angle size, from the very surprising Virgo cluster absence ( the most abundant nearby source at twenty Mpc), that the two hot spot, at 40-60 EeV, might be very probably related to lightest nuclei curier ( $\mathrm{He}, \mathrm{Be}, \mathrm{B}$, opaque at twenty Mpc) [14] and to a few near (few Mpc) active AGN as Cen A, M82 and NGC 253 ones[13].

3. We remark that the muon tracks by UHE hundred TeVs energy neutrinos are naturally the best tool for discovering the neutrino arrivals arrow, while cascades in icy are very poor in their directionality and they are almost a negligible contribute to any a neutrino astronomy.

4. Moreover above 30-60 TeVs muon tracks lenght in ice overcome several kilometers or even ten kilometers distances at hundred TeVs. These ten-kilometer sizes ranges forced us to consider widest, sponge like, structures, as the shell like spiral or the honey-comb ones, shown in present article.

5. Finally the present Icecube detector (a km cube volume) and future ones, at those hundred $\mathrm{TeV}$ energies, might include both the internal, HESE, (High Energy Starting Events), but also the more abundant and relevant external ones, the so called trought-going muons. These ones are greately enlarging the $v_{\mu}$ detection volume. These longest energetic tracks are at present a golden key to a more fruitfull Neutrino astronomy [15], while tau airshower neutrino astronomy still wait and grow in several future projects [8]. The best proposal up to day has been elaborated by a wide collaboration based on a twin orbital satellite detection 

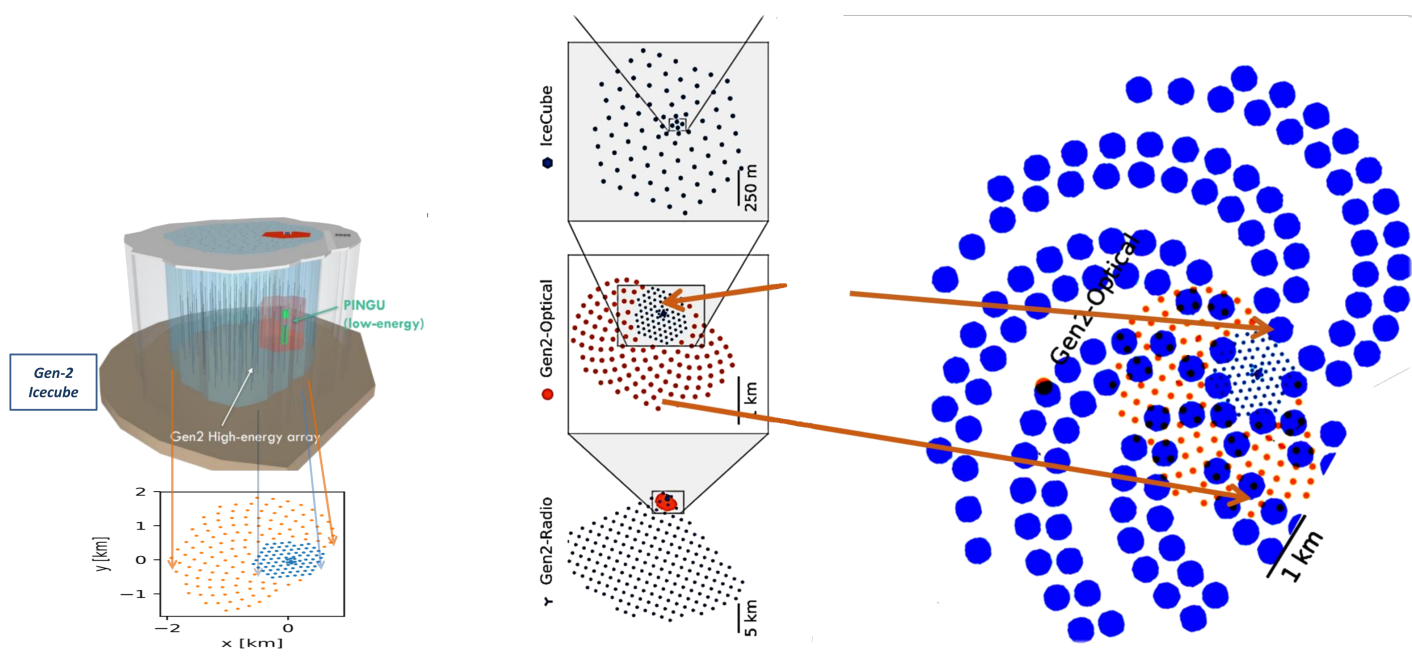

Figure 2: Left A representation of the "Ice Cube" architecture with a projection of the current effective volume with that achievable with the planned Gen-2 expansion. Right A representation of the IceCube architecture with a projection of the current effective volume and of that achievable with our planned Gen-2 expansion. An illustrative representation of a shell arrangement of the detectors, and the equivalent area obtainable. The figure offer an approximate comparison with the areas of the optical and radio Gen-2 upgrades extension. More details in the text.

(POEMMA) at Earth horizons or by a highest altitude balloons (Jem-EUSO) experiments [11].

\section{Homogeneous versus sponge like array as Shell-like or Honey-comb one}

The hundred $\mathrm{TeV}$ muon tracks as a neutrino astronomy are in competition with the promising upgoing tau airshower. At present days muon tracks in largest Icecube array remain the best tool we have for hundred TeVs or PeVs neutrino astronomy [15]. Therefore we focalize to our proposal to better expand the Gen-2 volume projects. Above hundred TeVs such external muons events, trough going ones, are more and more abundant respect internal ones, HESE, because their origination is in a much wider surrounding mass calorimeter; mainly rock below $2 \mathrm{~km}$ depth. Earth diameter becomes opaque to neutrinos at $30 \mathrm{TeVs}$ and energy above, while traveling across it. Therefore at hundred $\mathrm{TeV}$ s the most probable arrival signals are upgoing-horizontal ones, crossing minor terrestrial cords, leading to less or negligible neutrino opacity. Let us remind that future Gen-2 is imagined by Icecube collaboration, at present, by additional 120 strings spread in an homogeneous extende area, with a string to string common distance either as 240 or as 330 meter. [16]. We considered and preferred therefore only the horizontal highest energy tracks discover as the key to neutrino astronomy [15], suggesting as in figures, the widest geometrical configuration (sponge like ones), as a twin spiral arrays or a honey-comb ones, with several empty kilometer sized voids. Theses external and inner array geometry favor the widest volumes. These voids are loosing somehow the details of TeVs signals as well as the detection of short tracks and small size tens TeVs 

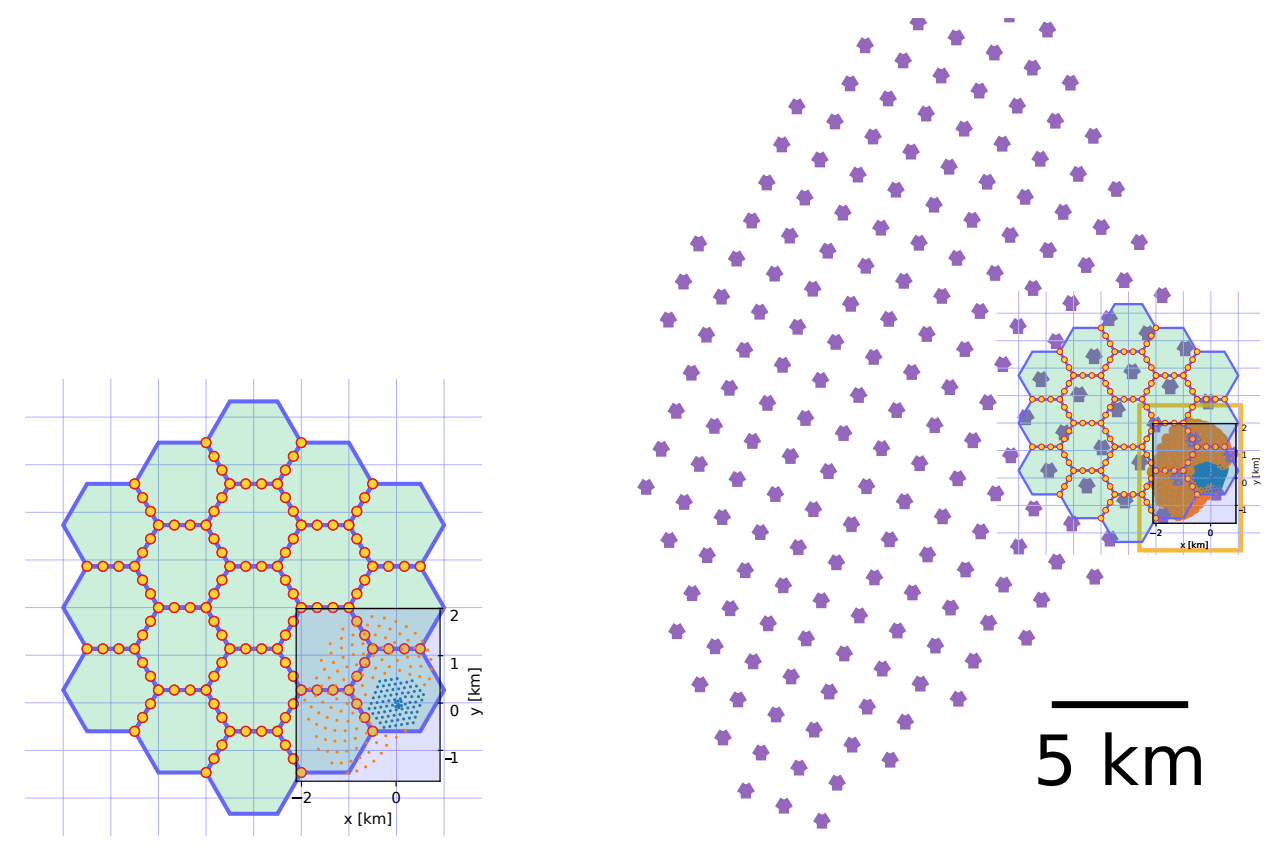

Figure 3: Left The Honeycomb design with $1 \mathrm{~km}$ wide side compared with the present Icecube and the Optical Gen-2 upgrade proposal for IceCube. Right The Honeycomb design compared with both the inner blue Icecube dot, the orange Gen-2 array area, the honey-comb proposal area as the project of the Radio Gen-2 upgraded area.

cascade or showers. Nevertheless the largest PeVs cascades, so volumetric, are anyway, mostly detectable by our present sponge array array.

\section{Conclusions}

Directionality in detection of multi-TeV energy astrophysical neutrinos in IceCube in correlation with detection of multi-TeV energy gamma in LHAASO experiment will provide the ideal multimessenger astronomical study of astrophysical sources of high energy gamma and neutrinos. In last decade muon tracks and cascades induced by tens or hundred TeVs neutrino opened a probable neutrino astronomy windows. The few hundreds cascade spherical signal are poor in directionality, while longest muon tracks events are the most accurate in arrival angles. The 2013 discover of a flavor ratio has been understood. Nowdays we need thousands of hundred $\mathrm{TeV}$ tracks to reach a statistical map with clustering, multi-plet, gamma-X-radio correlation. Such a rich map might be obtained not by a kilometer cube , but by a hundred kilometer track array detector. With present new 120 string array we imagined a best geometry structure as a sponge one, able to disentangle mainly longest meaningful muon tracks made by hundreds $\mathrm{TeV}$ primary astrophysicl neutrinos. These upward-horizontal signals might be HESE or trough-going ones. These longest kilometric 
tracks might cross the shell (or honey-comb) several sponge-like string net; they might capture even within such enlarged empty volumes, more and more directional track signals offering the hard, sharp as well as the most abundant neutrino sky map in very a near future.

\section{Acknowledgement}

The work by M.K. was supported by grant of the Russian Science Foundation (Project No-1812-00213-P).

\section{References}

[1] D. Fargion and P. Paggi, Flavor revolution at icecube horizons?, Nuclear Instruments and Methods in Physics Research Section A: Accelerators, Spectrometers, Detectors and Associated Equipment 753 (2014) 9-13.

[2] D. Fargion, P. Lucentini, M. Khlopov, P. Oliva, F. LaMonaca and P. Paggi, A charming IceCube discover?, in Proceedings of Frontier Research in Astrophysics - III PoS(FRAPWS2018), Sissa Medialab, Nov., 2019, DOI.

[3] D. Fargion, Discovering Ultra High Energy Neutrinos by Horizontal and Upward tau Air-Showers: Evidences in Terrestrial Gamma Flashes?, Astrophys. J. 570 (2002) 909 [astro-ph/0002453].

[4] D. Fargion, M. Grossi, M. De Santis, P. G. De Sanctis Lucentini, M. Iori, A. Sergi et al., Crown detectors to observe horizontal and upward air-showers, Adv. Space Res. 37 (2006) 2132 [astro-ph/0501079].

[5] D. Fargion, Arrays in space to detect upward tau and horizontal UHECR air showers, in International Cosmic Ray Conference, vol. 2 of International Cosmic Ray Conference, p. 903, Jan., 2001.

[6] D. Fargion, Horizontal and upward tau arishowers in Valleys, Mountains and Space: Discovering UHE neutrinos and new physics, in International Cosmic Ray Conference, vol. 3 of International Cosmic Ray Conference, p. 1297, Aug., 2001, astro-ph/0106239.

[7] D. Fargion, P. G. De Sanctis Lucentini and M. De Santis, Tau air showers from earth, Astrophys. J. 613 (2004) 1285 [hep-ph/0305128].

[8] D. Fargion, M. Grossi, M. De Santis and P. G. de Sanctis Lucentini, Rates of horizontal tau air-showers observable by satellites, in 35th COSPAR Scientific Assembly, 1, 2005, astro-ph/0501033.

[9] D. Fargion, D. D’Armiento, O. Lanciano, P. Oliva, M. Iacobelli, P. De Sanctis Lucentini et al., Splitting neutrino masses and showering into sky, Nuclear Physics B - Proceedings Supplements 168 (2007) 292-295. 
[10] D. Fargion, P. G. De Sanctis Lucentini, M. Grossi, M. De Santis and B. Mele, Ultrahigh-energy cosmic ray and UHE neutrino - relic-neutrino Z showering in dark halos, Mem. Soc. Ast. It. 73 (2002) 848 [hep-ph/0112014].

[11] M. Bagheri, P. Bertone, I. Fontane, E. Gazda, E. G. Judd, J. F. Krizmanic et al., Overview of Cherenkov Telescope on-board EUSO-SPB2 for the Detection of Very-High-Energy Neutrinos, PoS ICRC2021 (2021) 1191.

[12] T. P. A. Collaboration, Indication of a mass-dependent anisotropy above $10^{18.7} \mathrm{eV}$ in the hybrid data of the Pierre Auger Observatory, PoS ICRC2021 (2021) 321.

[13] D. Fargion, P. G. De Sanctis Lucentini and M. Y. Khlopov, Where are the Ultra High Energy Cosmic Ray (UHECR) originated?, PoS MULTIF2017 (2018) 006.

[14] D. Fargion, G. Ucci, P. Oliva and P. G. De Sanctis Lucentini, The meaning of the UHECR Hot Spots: A Light Nuclei Nearby Astronomy, EPJ Web Conf. 99 (2015) 08002 [1412 . 1573].

[15] D. Fargion, P. Oliva and P. D. S. Lucentini, Crossing muons in icecube at highest energy: a cornerstone to $v$ astronomy, Nuclear Physics B - Proceedings Supplements 256-257 (2014) 213 .

[16] IceCube-Gen2 collaboration, M. G. Aartsen et al., IceCube-Gen2: the window to the extreme Universe, J. Phys. G 48 (2021) 060501 [2008. 04323]. 